\title{
THE ECONOMICS OF MUTUALISMS: OPTIMAL UTILIZATION OF MYCORRHIZAL MUTUALISTIC PARTNERS BY PLANTS
}

\author{
Miroslav Kummel ${ }^{1,3}$ and Stephen W. Salant ${ }^{2}$ \\ ${ }^{1}$ Department of Environmental Science, Colorado College, Colorado Springs, Colorado 80907 USA \\ ${ }^{2}$ Department of Economics, University of Michigan, Ann Arbor, Michigan 48109 USA
}

\begin{abstract}
Can choice of mutualistic partners and the degree of their utilization determine (1) mutualistic partner coexistence, (2) relative abundance of mutualistic partners, and (3) environment-dependent changes in relative abundance? We investigate these questions in the context of the plant-mycorrhizal fungal mutualism by building a biological market model potentially applicable to other mutualisms as well. We examine the situation where a single plant selectively utilizes member(s) of a group of ectomycorrhizal potential trading partners. Under biologically realistic circumstances, the plant may simultaneously utilize multiple partners, its degree of utilization determining the community structure of the fungi. If utilization of multiple partners is optimal, the marginal cost of acquiring additional nitrogen from every trading partner must be equal while the marginal cost of acquiring it from any unutilized partner must be larger. Because the plant's nitrogen demand is light dependent, the composition of the fungal species among its trading partners changes along light-availability gradients. We discuss the design of an experiment to test the key prediction of our model, the equalization of marginal cost.
\end{abstract}

Key words: biological market; marginal cost; mutualism; mycorrhizae; optimal choice; partner choice.

\section{INTRODUCTION}

Mutualisms are ubiquitous in nature (Janzen 1985), with a significant portion of mutualistic interactions involving associations with multi-species mutualistic partner communities (reviewed by Stanton 2003). For example, a single plant is commonly associated with several species of mycorrhizal fungal partners (Smith and Read 1997). There are two unique attributes of these partner communities. First, the quality of the partners in the mutualistic partner communities is documented to vary from good mutualists, to mediocre mutualists, to cheaters that appear to provide nothing in return for the rewards they reap (Stanton 2003). Second, in addition to potential direct partner-partner interactions, the mutualistic partners interact with one another indirectly through their direct interactions with the shared host mutualist. Thus, the host, through its morphological, physiological, and behavioral responses to the partners, can potentially play a key role in regulating the partner community. These responses of the host can lead to favoring or "choosing" certain partners over others. The goal of this paper is to examine the potential role of this "partner choice" in mediating partner coexistence, determining relative abundances of partners, and shifting relative abundances of partners with shifting resources-in a system marked by an ecologically significant variation of partner quality.

Manuscript received 20 February; revised 29 August 2005; accepted 2 September 2005. Corresponding Editor: D. A. Wardle.

${ }^{3}$ E-mail: mkummel@coloradocollege.edu
The concept of partner choice was applied to the study of mutualisms already by Darwin (1859, in Sachs et al. 2004) in his explanation of the evolution of nectaries. Since then partner choice gained popularity in the literature on both interspecific and intraspecific cooperation (see the review by Sachs et al. 2004); early examples include mate choice (Dawkins 1976), the titfor-tat strategy of the iterated prisoner's dilemma, the evolutionary stability of tit-for-tat, and establishment of tit-for-tat in a defector population (Axelrod and Hamilton 1981, Eshel and Cavallisforza 1982). Empirical observation of baboon interactions led Noë (1990) to take partner choice out of the iterative framework, and Bull and Rice (1991) proposed that partner choice alone could lead to the evolution and maintenance of mutualism - an idea that is currently receiving both empirical and theoretical attention (Denison 2000, Kiers et al. 2003). The verbal theory of biological markets (Noë and Hammerstein 1994) expanded the concept of partner choice from a dichotomous decision to the choice of the extent of partner utilization-an idea central to our paper.

To operate, partner choice requires variation in partner quality, assessment of partner quality by the host, and a decision rule to determine which partners to associate with, and on the degree of association with each chosen partner (West et al. 2002). The decision rule also involves morphological, physiological, or behavioral mechanism that encourages the association of the host with partners contributing to greater reproductive success and association of the host with low-quality partners (Sachs et al. 2004). This flexible strategy leads 
to the choice of more beneficial individual partners. However, if the interspecific variation in costs and benefits associated with individual partners is greater than the intraspecific variation, then this choice of individuals necessarily leads to consistent and predictable choice of more beneficial species of partner mutualists. Over evolutionary time the hosts may evolve fixed preferences for or against certain species of partners, and thus replace the assessment-decision rule system with a partner-species-recognition system.

Partner choice is likely to be a common phenomenon in nature. In a recent review of the game-theoretical structure of mutualistic systems, Bshary and Bronstein (2004) examined the potential for partner choice across different mutualisms and concluded that with the exception of vertically transmitted mutualisms, all other classes of mutualisms exhibited the potential features of partner choice. Well-documented cases of partner choice include pollination syndromes of plants (Bshary and Bronstein 2004), choice of plant species by pollinators (Bshary and Bronstein 2004), choice of aphid species by ants (Fischer et al. 2001), choice of luminescent bacteria strains by squids (Visick et al. 2000 in Sachs et al. 2004), and sanctions of legumes against cheating Rhizobia (Denison et al. 2003, Kiers et al. 2003). Selective carbon allocation by plants toward better performing mycorrhizal fungi is currently under investigation (J. D. Bever, personal communication).

The degree to which partner choice determines partner species coexistence and relative abundance of partner species is largely unknown. In some systems partner choice is not necessary for partner species coexistence - as shown by coexistence of a superior mutualistic partner and an inferior mutualistic partner (or a cheater) in models of Bronstein et al. (2003), and Ferriere et al. (2002), none of which includes partner choice. However, in other systems, such as the model of Hoeksema and Kummel 2003, partner choice is necessary for partner coexistence. Currently, there is growing empirical interest in the role that partner choice plays in shaping partner population and community dynamics. For example the choice of a more desirable aphid species draws ant protection away from the less desirable aphid species which, as a result, experience a higher level of predation and population decline (Fischer et al. 2001). Partner choice is likely to be an important driving force in systems that resemble biological markets (Noë and Hammerstein 1994). Mutualisms are based on a mutually beneficial exchange of resources or services (Bronstein 1994). However, specific conditions have to be met for this exchange to resemble trading within a biological market. These conditions include exchange of commodities, choice of trading partners, and competition via outbidding (Noë and Hammerstein 1994). Clearly, not all mutualistic systems fit this description. However, in the systems that do satisfy the assumptions of biological markets, the use of economic analogy and the tools of economic analysis is fully appropriate.
The goal of this paper is to provide the first formal mathematical model of the role of biological markets in structuring communities of mutualistic partners associated with individual host mutualists. We investigate the following questions: Can partner choice, via differential partner utilization, lead to coexistence of several mutualistic partners on a single host? Can partner choice determine community structure of the mutualistic partners, and can it explain changes in that community structure in response to changing resource availability to the host?

To address these questions, we build a model where the growth of a host mutualist is dependent on the resources it has available after trading with its diverse mutualistic partners. Each potential partner is represented by its exchange function, which relates the amount of resource 1 that the host must relinquish as a payment for any given amount of resource 2 delivered by that partner (e.g., how much carbon a plant needs to relinquish in exchange for any given amount of nutrient delivered by a mycorrhizal fungus). The host mutualist selects the extent of use of its potential trading partners to maximize its growth.

We assume that the host and partner mutualists engage in an exchange of resources, that the same sets of resources are exchanged between the host mutualist and each of the partners, that the amount of resource 1 acquired per unit of resource 2 relinquished (the "price") may vary among the partners, and that the host mutualist can adjust the degree of utilization of its partners. We initially assume that the partner mutualists do not interact with one another but we subsequently relax this simplification.

\section{The Biological System}

Our investigation focuses on symbiotic mutualistic systems where one host mutualist simultaneously interacts with several partner mutualists although it can be generalized easily to the case where there are multiple host mutualists (unpublished analysis available from the authors). Biological examples include plants interacting with several mycorrhizal fungi (Smith and Read 1997), legumes hosting several genotypes of nitrogen-fixing bacteria (Denison 2000), and corals hosting several genotypes of zoozentele (Knowlton and Rohwer 2003). Our model could be illustrated by any symbiotic mutualism that conforms to the biological markets assumptions (Noë and Hammerstein 1994); however, for concreteness we developed the model in the context of the mutualism between plants and ectomycorrhizal fungi. The central feature of this mutualism is an exchange of carbon assimilated by the plant for nutrients (chiefly nitrogen) taken up by the fungi from the soil (Smith and Read 1997). Individual plants simultaneously associate with several species of ectomycorrhizal fungi (5-15 species of fungi commonly associate with individual balsam fir seedlings, [M. Kummel, personal observation]), and ectomycorrhizal 

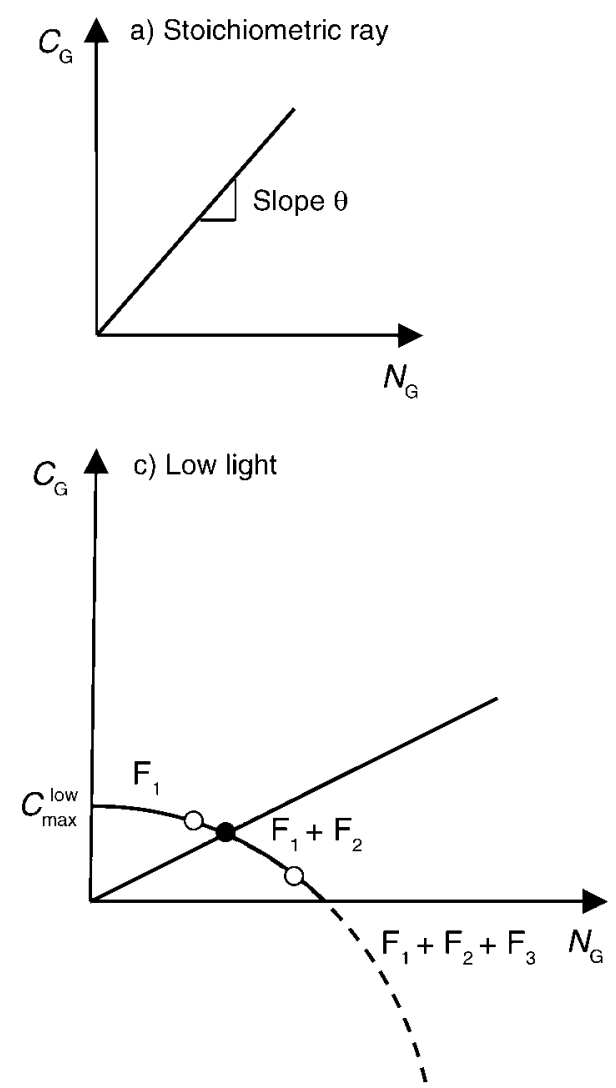
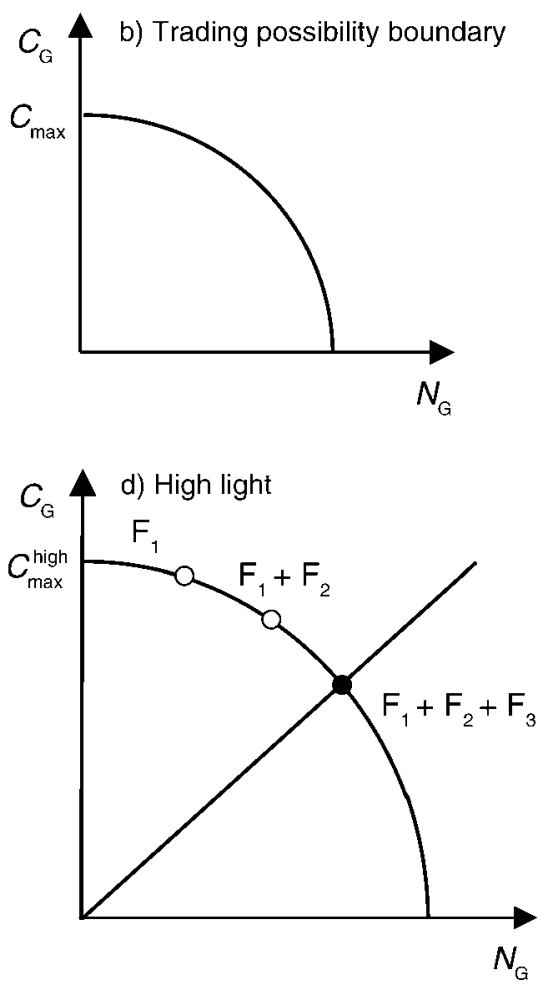

FIG. 1. (a) The stoichiometric ray and (b) the trading possibility boundary. The total nitrogen acquired and total carbon remaining is represented by the two coordinates of the point of intersection of the ray and the boundary. $C_{\mathrm{G}}$ represents the amount of carbon retained for growth, $N_{\mathrm{G}}$ represents the amount of nitrogen retained for growth, and $C_{\max }$ is the plant's total units of carbon (its photosynthetic potential. An increase in the light level from (c) low to (d) high shifts every point on the trade possibility boundary up vertically by $C_{\max }^{\text {high }}-C_{\max }^{\text {low }}$ (where $C_{\max }^{\text {low }}$ is the photosynthetic potential at low light and $C_{\max }^{\text {high }}$ is the photosynthetic potential at high light) and consequently results in a new intersection point farther out along the ray. In the low-light case (c) the plant uses fungi $F_{1}$ and $F_{2}$; in the high-light case (d), the plant acquires more nitrogen not only by utilizing $F_{1}$ and $F_{2}$ more intensively, but also by adding $\mathrm{F}_{3}$ as a third trading partner.

fungi vary in their carbon for nutrient exchange rates (Jones et al. 1998). Plants are likely to have the capacity to choose the composition of ectomycorrhizal partners through either selective root turnover or through selective allocation of carbon to roots harboring more effective species of fungi (Janos 1985).

\section{THe Model}

Consider a plant of unit size, which maximizes its aboveground rate of growth. To grow, the plant requires both $\mathrm{C}$ and $\mathrm{N}$ in the fixed ratio $\theta$, which reflects the $\mathrm{C}$ and $\mathrm{N}$ stoichiometry of building new tissues. We represent the plant's growth requirements by a ray from the origin in $\mathrm{C}-\mathrm{N}$ space with positive slope $\theta$ (Fig. 1a). Points further out along the ray generate faster growth. We refer to this ray, which plays an important role in our analysis, as the "stoichiometric ray."

The plant assimilates carbon in photosynthesis and uses some portion to purchase nitrogen from associated mycorrhizal fungi. We assume that if the plant relinquishes more carbon to a given fungus, it always receives more nitrogen in exchange. The plant is endowed with $C_{\max }$ units of carbon (its photosynthetic potential). A portion of this carbon $\left(C_{\mathrm{N}}\right)$ is traded to the mycorrhizal fungi in exchange for nitrogen while the remainder $\left(C_{\mathrm{G}}\right)$ is retained for growth: $C_{\max }=C_{\mathrm{G}}+C_{\mathrm{N}}$. In exchange for its aggregate payment of $C_{N}$ units of carbon to the ectomycorrhizal fungi, the plant receives in aggregate $N_{\mathrm{G}}$ units of nitrogen.

To maximize growth, the plant relinquishes as little carbon $\left(C_{\mathrm{N}}\right)$ as possible in exchange for any given amount of nitrogen. How much carbon the plant relinquishes depends on (1) the mycorrhizal fungi selected by the plant and (2) the intensity with which it utilizes each fungus.

\section{How a plant utilizes multiple partners to maximize growth}

We will assume that each species of mycorrhizal fungus is endowed with its own exchange function, $C_{N f}=$ $r_{f}\left(N_{f}\right)$, which we treat as given. Fungus $f($ for $f=1, \ldots, F)$ 
requires $r_{f}\left(N_{f}\right)$ units of carbon in order to provide $N_{f}$ units of nitrogen. We assume that the plant relinquishes carbon if and only if it receives nitrogen in return and to acquire more nitrogen it must relinquish more carbon. Hence, we assume each exchange function passes through the origin and is increasing. To permit the use of calculus, we also assume each exchange function is twice differentiable for $N_{f}>0$. For each aggregate amount of nitrogen acquired $\left(N_{\mathrm{G}}\right)$, the plant is assumed to utilize the available fungi to minimize its aggregate carbon "payment." Denote this minimized payment as $H\left(N_{\mathrm{G}}\right)$. Formally,

$$
H\left(N_{\mathrm{G}}\right)=\min _{\left\{N_{f}\right\}} \sum_{f=1}^{F} r_{f}\left(N_{f}\right)
$$

subject to

$$
\sum_{f=1}^{F} N_{f}=N_{\mathrm{G}}
$$

and

$$
N_{f} \geq 0 \quad \text { for } f=1, \ldots, F .
$$

After paying for the nitrogen acquired in the cheapest way, the plant retains $C_{\mathrm{G}}=C_{\mathrm{max}}-H\left(N_{\mathrm{G}}\right)$ units of carbon. We refer to this decreasing function, which plays a leading role in our analysis, as the trading possibility boundary (Fig. 1b). The carbon retained after payment, when combined with the nitrogen acquired from the trading partners, determines the plant's growth. The aggregate nitrogen acquisition that maximizes the plant's growth is determined graphically as the horizontal component of the point of intersection of the stoichiometric ray and the trading possibility boundary (Fig. 1c and 1d).

The curvature of the exchange functions available to the plant determines whether the plant has a single trading partner or several trading partners. It also determines the curvature of the trading possibility boundary. We begin with the case where there are only two fungi of different species $(F=2)$ available to the plant. This case is simplest and is used as a building block in the more general case we discuss, where $F \geq 2$.

To acquire $N_{\mathrm{G}}$ units of nitrogen at lowest cost from two species of fungus, the plant minimizes the following function of one variable: $g\left(N_{1}\right) \equiv r_{1}\left(N_{1}\right)+r_{2}\left(N_{\mathrm{G}}-N_{1}\right)$, where $N_{1} \in\left[0, N_{\mathrm{G}}\right]$. If the global minimum occurs at $N_{1}=$ 0 , the plant utilizes only fungus 2 and $g^{\prime}(0) \geq 0$; for if $g^{\prime}(0)<0$, more reliance on fungus 1 would reduce costs. If the global minimum occurs at $N_{1}=N_{\mathrm{G}}$, the plant utilizes only fungus 1 and $g^{\prime}\left(N_{\mathrm{G}}\right) \leq 0$; for if $g^{\prime}\left(N_{\mathrm{G}}\right)>0$, less reliance on fungus 1 would reduce costs. If the plant utilizes both fungi simultaneously, then $N_{1} \in\left(0, N_{\mathrm{G}}\right)$ and the standard results from introductory calculus imply that the following pair of conditions must hold:

$$
\begin{aligned}
& g^{\prime}\left(N_{1}\right) \equiv r_{1}^{\prime}\left(N_{1}\right)-r_{2}^{\prime}\left(N_{\mathrm{G}}-N_{1}\right)=0 \\
& g^{\prime \prime}\left(N_{1}\right) \equiv r_{1}^{\prime \prime}\left(N_{1}\right)+r_{2}^{\prime \prime}\left(N_{\mathrm{G}}-N_{1}\right) \geq 0 .
\end{aligned}
$$

Following the short-hand terminology in economics, we refer to the additional cost of acquiring another unit of nitrogen from fungus $f$ when that fungus is providing $N_{f}$ units of nitrogen (the first derivative $r_{f}^{\prime}\left(N_{f}\right)$ ) as the "marginal cost." Condition 1 says that whenever the plant utilizes both fungi simultaneously, their marginal costs must be equal. We refer to the value of marginal cost that is equalized among the fungal trading partners $\left(r_{1}^{\prime}\left(N_{1}\right)=r_{2}^{\prime}\left(N_{\mathrm{G}}-N_{1}\right)\right)$ as the "common marginal cost."

Condition 2 says that if the plant utilizes both fungi, the sum of the slopes of the two marginal-cost functions cannot be negative. For suppose the two marginal costs are equal but $r_{1}^{\prime \prime}\left(N_{1}\right)=r_{2}^{\prime \prime}\left(N_{\mathrm{G}}-N_{1}\right)<0$. This inequality requires, of course, that at least one of the two exchange functions is concave $\left(r_{f}^{\prime \prime}\left(N_{f}\right)<0\right)$. Then since $g^{\prime \prime}\left(N_{1}\right)<0$, carbon costs would be at a relative maximum and, while no local variation in $N_{1}$ could yield a first-order reduction in the carbon payment, non-local variations in $N_{1}$ in either direction would reduce costs.

\section{Exchange functions of differing shapes and their consequences}

It is useful to consider how carbon payments would be minimized if every exchange function available is concave or linear ( $r^{\prime \prime}<0$ or $r^{\prime \prime}=0$, respectively) on the one hand or convex $\left(r^{\prime \prime}>0\right)$ on the other. This will facilitate understanding the more general case where a mixture of exchange functions of these three shapes is available to the plant.

Concave and linear exchange functions $\left(r^{\prime \prime} \leq 0\right)$.Suppose the plant has available two distinct species of fungi, each with a different linear or concave $\left(r^{\prime \prime} \leq 0\right)$ exchange function. Then to obtain $N_{\mathrm{G}}$ units of nitrogen, the plant will trade with only one of the fungi. For, if it were optimal to obtain nitrogen from both of them simultaneously, condition 1 and 2 of the previous subsection must hold and this is impossible: if the two fungi have distinct linear exchange functions, then one fungus provides nitrogen at a lower marginal cost than the other and condition 1 cannot be satisfied; if instead one fungus has a concave exchange function and the other has a linear or concave function, then even if both fungi provide nitrogen at the same marginal cost, the sum of the second derivatives of their exchange functions is negative and condition 2 is violated.

To obtain a given amount of nitrogen from two fungi with linear or concave exchange functions, therefore, the plant will rely on only one of them at a time. But the plant may switch allegiance from one fungus to the other if it needs a different amount of nitrogen. In Fig. 2a, the linear exchange function is below the concave function to the left of the intersection point and above it to the right. The plant, therefore, uses the fungus with the linear exchange function when the plant's nitrogen needs are low but switches to the fungus with the concave exchange function when its nitrogen needs are high. In Fig. 2b, each fungus has a concave exchange function and they intersect twice. In this case, as its nitrogen 

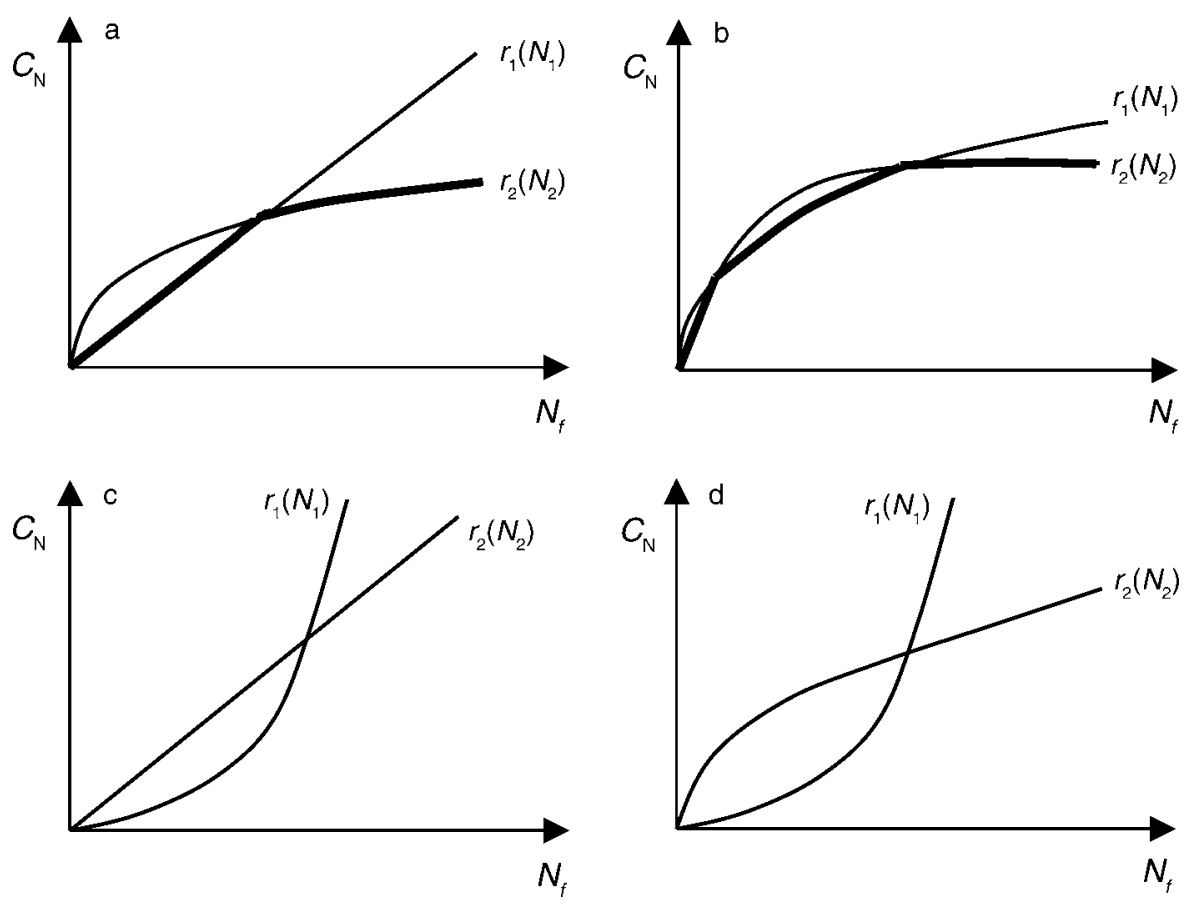

FIG. 2. Only one fungus from a set of available fungi will be utilized if all have linear or concave exchange functions as in panels (a) and (b). The smallest carbon payment needed to acquire any specified amount of nitrogen will be given by the lower envelope of the exchange functions (the heavy line). Although (a) and (b) depict cases with two linear or concave exchange functions, the same results hold with any number of functions with this curvature. Panels (c) and (d) depict cases with one strictly convex exchange function and the other weakly concave. In such cases, the plant may utilize both partners (at equal marginal cost) and, in brighter light, would rely more on the concave fungus. Fig. 3 treats the case where all exchange functions are instead convex. A final case occurs when some functions are convex and the others are linear or concave. To derive the trading possibility boundary in this case, first construct the lower envelope of all the linear or concave exchange functions as in Fig. 2, panels (a) and (b), and then use this single weakly concave "pseudo exchange function" (the lower envelope) along with the set of convex exchange functions in the procedure in Fig. 3.

needs increase, the plant switches from one fungus to the other at the first intersection point but returns to the original fungus beyond the second intersection point. Notice that in each of these examples, the minimum carbon payment for any nitrogen need, $X$, is simply the lower envelope of the two exchange functions. Denote this minimum payment as $H_{\mathrm{v}}(X)$. Like the exchange functions which make up each segment, this lower envelope $H_{\mathrm{v}}(X)$ is increasing and either linear or concave. However, whenever the plant switches to a different fungus, the lower envelope may be kinked. At each kink, the right derivative is smaller than the left derivative (but positive) because the exchange function of the replacement trading partner is below that of the fungus which is being displaced. Hence, $H_{\mathrm{v}}(X)$ is weakly concave (concave or linear) throughout.

All of these properties generalize if there are many fungi with linear or concave exchange functions. The plant never uses more than one of them at a time. The carbon cost of obtaining $X$ units of nitrogen in the cheapest way is again, therefore, the lower envelope of the exchange functions and this envelope $\left(H_{\mathrm{v}}(X)\right)$ is increasing, may be kinked at switch points, and weakly concave throughout.
The carbon available for growth after paying $H_{\mathrm{v}}(X)$ out of the photosynthetic potential $\left(C_{\max }\right)$ is simply $C_{\mathrm{G}}$ $=C_{\max }-H_{\mathrm{v}}(X)$. Given the properties of $H_{\mathrm{v}}(X)$, this "trading possibility boundary" is downward sloping, weakly convex, and may be kinked.

Convex exchange functions $\left(r^{\prime \prime}>0\right)$. - Suppose instead the plant has available two distinct exchange functions that are convex. This shape raises the possibility that coexistence condition 1 is satisfied and ensures that the sum of second derivatives is strictly positive, satisfying coexistence condition 2. Let fungus $1^{\prime} \mathrm{s}$ exchange function have the smaller marginal cost at the origin $\left(r_{1}^{\prime}(0)<r_{2}^{\prime}(0)\right)$. Then, if its nitrogen needs are low, the plant may rely entirely on fungus 1 . As its nitrogen needs increase, the plant will rely more intensively on fungus 1 and, since its exchange function is convex, its marginal cost will increase. Eventually a threshold $(\hat{X})$ is reached where $r_{1}^{\prime}(\hat{X})=r_{2}^{\prime}(0)$. If more than $\hat{X}$ units of nitrogen are needed, the plant must then take on fungus 2 as an additional trading partner in order to minimize costs. Since the plant is then utilizing two sources at the same time, the plant must acquire nitrogen from each source so that the marginal costs are equal. As the plant acquires more nitrogen in aggregate, the common 
marginal cost rises and each trading partner is used more intensively. Denote the minimized cost of obtaining $X$ units of nitrogen from the convex exchange functions as $H_{\mathrm{c}}(X)$. There is zero cost if no nitrogen is obtained and higher cost if more is obtained. Hence, $H_{\mathrm{c}}(X)$ is increasing and passes through the origin. When the nitrogen demand is low, fungus 1 is relied upon exclusively and $H_{\mathrm{c}}(X)$ is convex since it coincides with $r_{1}(X)$. After fungus 2 becomes a trading partner, if the plant needs additional nitrogen it must exploit each partner more intensively so that the marginal costs remain equalized and this raises their common value. Since the slope of $H_{\mathrm{c}}(X)$ at any point where there are multiple trading partners is equal to the common marginal cost underlying that point, $H_{\mathrm{c}}(X)$ is convex. At the point $\hat{X}$ where the plant's nitrogen needs are just sufficient to bring in the second fungus, the additional cost of acquiring slightly more nitrogen is $r_{1}^{\prime}(\hat{X})$ and the cost saving of acquiring slightly less nitrogen is also $r_{1}^{\prime}(\hat{X})$. Hence, there can be no kink at $\hat{X} . H_{\mathrm{c}}(X)$ is increasing, smooth, and convex throughout.

All of this generalizes easily if the plant has available any number of fungi with convex exchange functions. In that case, the plant may rely on one fungus or on several. If several, the plant obtains nitrogen from each of its trading partners in such a way that the marginal costs are equated (as condition 1 requires). If the plant obtains more nitrogen in aggregate, the common marginal cost will increase and each pre-existing trading partner will provide more nitrogen. If costs are minimized, any available fungus with $r_{f}^{\prime}(0)$ smaller than this common marginal cost must be included as a trading partner. When all the fungi available to the plant have convex exchange functions, each fungus has "job security": once the plant accepts it as a trading partner, it will never get "laid off" or even utilized less intensively as long as the plant's nitrogen needs increase. A graphical technique for predicting which fungi from a set with convex exchange functions will be included as trading partners and how intensively each will be utilized in order to acquire any specified aggregate amount of nitrogen is provided in Fig. 3 .

Once again, the carbon available for growth after paying $H_{\mathrm{c}}(X)$ to obtain $X$ units of nitrogen is simply $C_{\mathrm{G}}$ $=C_{\max }-H_{\mathrm{c}}(X)$. Given the properties of $H_{\mathrm{c}}(X)$, this trading possibility boundary is downward sloping, concave, and smooth.

Mixtures of convex $\left(r^{\prime \prime}>0\right)$ and linear $\left(r^{\prime \prime}=0\right)$ or concave $\left(r^{\prime \prime}<0\right)$ exchange functions. - Suppose instead the plant has available two distinct species of fungi: fungus 1 with a convex exchange function and fungus 2 with a linear or concave exchange function. As before, each exchange function is assumed to pass through the origin and to be smooth and increasing $\left(r^{\prime}>0\right)$. If $r_{1}^{\prime}(0)$ $\geq r_{2}^{\prime}(0)$, the plant never uses fungus 1 . But suppose $r_{1}^{\prime}(0)$ $<r_{2}^{\prime}(0)$ as in Fig. 2c and 2d. Then the plant relies exclusively on fungus 1 for small amounts of nitrogen. But as the plant needs more nitrogen, fungus 1 provides it on increasingly unfavorable terms. At some point, fungus 2 will look attractive as an additional trading partner. This happens at the threshold where the plant must acquire $\tilde{X}$ units of nitrogen in aggregate, where $r_{1}(\tilde{X})=r_{2}^{\prime}(0)$. If the plant needs more than $\tilde{X}$ units of nitrogen, it includes fungus 2 as a trading partner. Since it then is using two sources simultaneously, the two marginal costs must have a common value. There are two cases to consider, illustrated in Fig. 2.

In the first case (Fig. 2c), the exchange function of fungus 2 is linear. Then for aggregate nitrogen acquisition to increase while at the same time the two marginal costs remain equal, the plant must obtain all of the additional nitrogen from the linear exchange function of fungus 2 and must not alter its acquisition from the convex exchange function of fungus 1 . In this case, the common marginal cost at the two sources remains equal to the constant slope of the linear exchange function.

In the second case (Fig. 2d), the exchange function of fungus 2 is concave. If the plant's nitrogen needs $\left(N_{\mathrm{G}}\right)$ are increased and it continues to utilize both fungi, then conditions 1 and 2 must continue to hold. Since drawing more nitrogen from fungus 1 raises its marginal cost while acquiring more from fungus 2 lowers its marginal cost, the plant must increase its acquisition of nitrogen from one fungus while reducing its acquisition from the other in order to maintain the equality of the marginal costs (condition 1). But which fungus does the plant rely on more heavily for the additional nitrogen? Condition 2 requires that if the plant needs more nitrogen, it relies increasingly on the fungus with the concave exchange function and reduces (although to a lesser extent) its utilization of the fungus with the convex exchange function, thereby reducing the common marginal cost. This result makes intuitive sense: when the plant needs more nitrogen, it relies more heavily on the fungus which gives it "discounts" $\left(r_{2}^{\prime \prime}\left(N_{2}\right)<0\right)$ on additional quantities and less on the fungus which gives it "surcharges" $\left(r_{1}^{\prime \prime}\left(N_{2}\right)>0\right)$ for additional quantities.

All of this generalizes easily if the plant has available any number of exchange functions some of which are convex and the remainder are linear or concave. We continue to assume that all exchange functions are smooth, increasing and pass through the origin. In this more general case, what would the plant do to minimize the cost of obtaining $N_{\mathrm{G}}$ units of nitrogen? Denote as $X$ $\in\left[0, N_{\mathrm{G}}\right]$ the nitrogen the plant obtains from the set of convex fungi and $N_{\mathrm{G}}-X$ as the nitrogen it obtains from the set of linear or concave fungi. Whatever nitrogen the plant obtains from each group must be acquired in the cheapest way if carbon costs are to be minimized. To obtain any amount of nitrogen from the set of linear or concave fungi in the cheapest way, the plant will rely entirely on one fungus. To obtain any amount of nitrogen from the set of fungi with convex exchange functions ("convex fungi"), the plant will utilize one or more of them. If it obtains $X \in\left[0, N_{\mathrm{G}}\right]$ units of nitrogen 

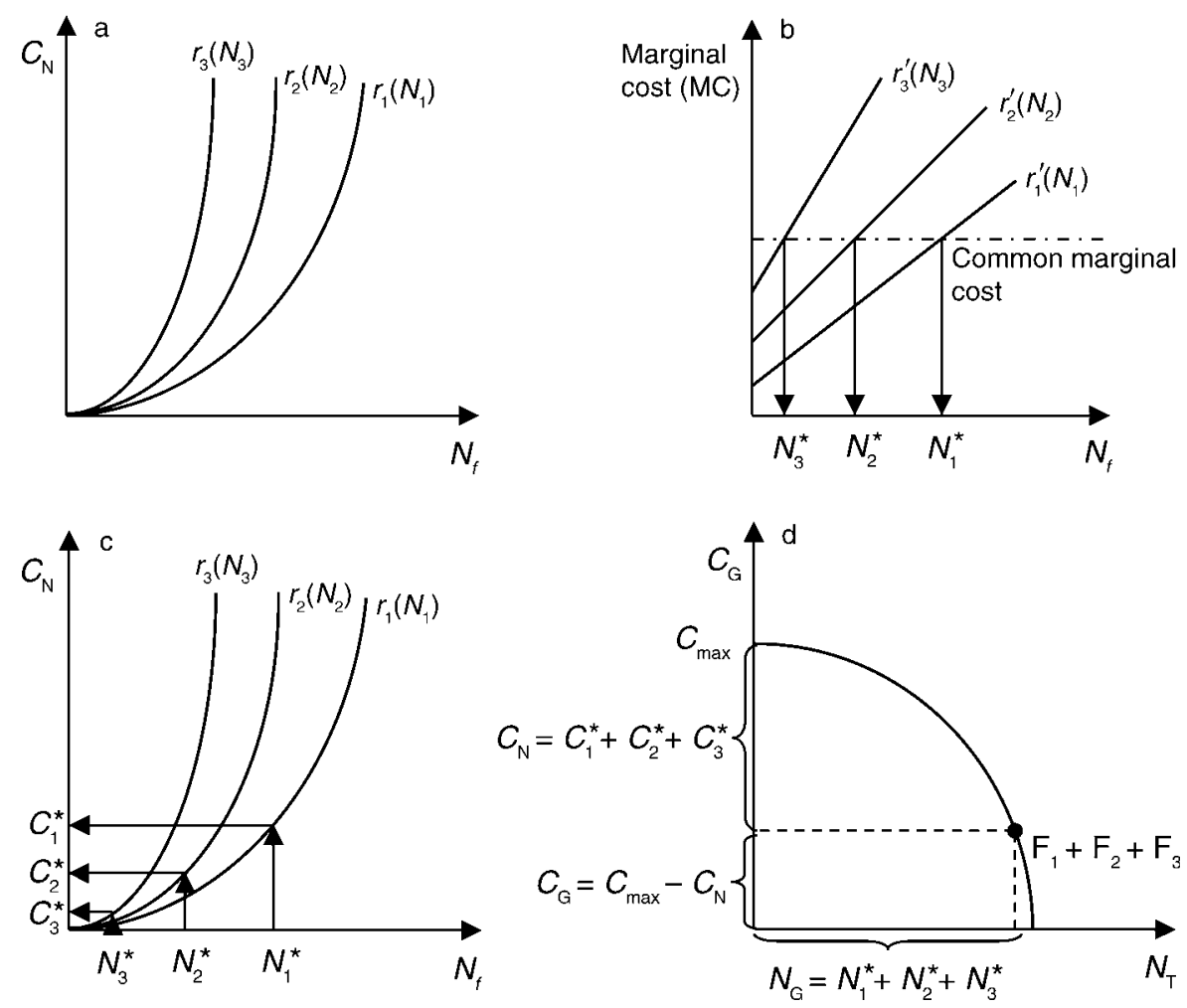

FIG. 3. (a) Three exchange functions and (b) the associated marginal-cost curves. To determine the minimal carbon payment needed to obtain a specified $N_{\mathrm{G}}$, adjust the horizontal common marginal-cost line in panel (b) until the horizontal distance between the vertical axis and the point where each marginal-cost curve intersects the common marginal-cost line (measuring the nitrogen acquired from each fungus) adds up to the specified $N_{\mathrm{G}}$. Panel (c) shows how to determine the carbon payment to each fungus while (d) associates the specified horizontal component $N_{\mathrm{G}}$ with the computed vertical component $C_{\mathrm{G}}$ to produce one point on the trade possibility boundary. Repeating this procedure with different $N_{\mathrm{G}}$ sweeps out the entire boundary. This procedure can be used for any number of convex exchange functions and can be easily modified as described in The model: Extensions if some are linear or concave. $N_{f}^{*}$ is the optimal amount of nitrogen acquired from fungus $f$ (i.e., $N_{1}^{*}$ is the optimal amount of nitrogen acquired from fungus 1 ); $C_{f}^{*}$ is the payment of carbon the plant has to make to fungus $f$ for acquiring $N_{f}^{*}$ units of nitrogen.

from the convex fungi and the remainder from the linear or concave fungi, the carbon cost will be $H_{\mathrm{c}}(X)+$ $H_{\mathrm{v}}\left(N_{\mathrm{G}}-X\right)$. To maximize the plant's growth, $X$ should be chosen to minimize this cost. Denote the minimized cost as $H\left(N_{\mathrm{G}}\right)$, where

$$
H\left(N_{\mathrm{G}}\right)=\min _{X \in\left[0, N_{\mathrm{G}}\right]} H_{\mathrm{c}}(X)+H_{\mathrm{v}}\left(N_{\mathrm{G}}-X\right) .
$$

This minimized carbon payment is zero if no nitrogen is acquired and increases as more nitrogen is acquired. If it is advantageous for the plant to obtain even the first unit of nitrogen from a linear or concave fungus, then all subsequent units will also come from some linear or concave fungi. However, if the first unit of nitrogen comes from a convex fungus or a group of convex fungi, then if nitrogen needs are sufficiently large, a threshold may be reached where a single linear or concave fungus is included in the set of trading partners along with these convex fungi. If more nitrogen is then required and the new fungus is linear, the plant would hold its utilization of the convex fungi constant and increase its utilization of the linear fungus. If the new fungus is concave, then the plant would scale down its utilization of the convex fungi (perhaps dumping a few) as it is increasing the utilization of the concave fungus. In either case, as the nitrogen demand increases new thresholds might be crossed beyond which the plant replaces the lone linear or concave fungus among its trading partners with a different lone linear or concave fungus.

Given the properties of $H\left(N_{\mathrm{G}}\right)$, the trading possibility boundary is downward sloping, smooth, and concave as long as every trading partner has a convex exchange function. In ranges where a lone concave or linear exchange function is also utilized, the trading possibility boundary may be kinked with each segment linear if the lone exchange function utilized in that range is linear and convex otherwise.

\section{Theory and preliminary evidence about the curvature of fungal exchange functions}

Because our model may be applicable to other mutualisms with exchange functions of various shapes, we have explored in some depth the consequences of alternative curvature assumptions. However, theoretical 
arguments suggest that in the case of fungi, the exchange functions are convex $\left(r^{\prime \prime}>0\right)$. This shape should result from morphological or physiological specialization of the mycelium into mycelial fans that actively forage and transport hyphae that do not forage, but require carbon for maintenance. Increasing amounts of nitrogen requested from the fungus need to be transported from increasing distances, thus leading to disproportionately larger amounts of carbon allocated to transport. This disproportionate increase in nitrogen transport cost would drive up the cost of every additional unit of nitrogen requested. Not only does the hypothesis that exchange functions are convex seem to us theoretically sensible but it is consistent with the admittedly limited experimental evidence currently available (Jones et al. 1991).

We also assume that the exchange functions pass through the origin. Given the complete lack of empirical data on the subject, this assumption is a reasonable "null hypothesis." A negative carbon intercept implies that the fungus pays the plant carbon in order for the plant to accept its nitrogen, a highly unlikely scenario. A positive carbon intercept, on the other hand, would indicate that the fungus requires a "minimum payment"-perhaps related to its basal metabolic rate-before it relinquishes any nitrogen. We explore the consequences of convex exchange functions with positive intercepts elsewhere (unpublished analysis available from the authors) and find that partner coexistence still requires equalization of marginal costs. In this case, however, the trading possibility boundary is not concave since it has concave segments separated by kinks where the right derivative must have the flatter slope. The kinks correspond to nitrogen uptake thresholds where the plant is predicted to scale down or discontinue utilization of low-initial-payment, high-initial-marginal-cost fungi and switch to using high-initial-payment, low-initial-marginal-cost fungi.

\section{Extensions}

We extend our model to two different situations. First we consider how the utilization of the mycorrhizal fungi changes with increasing resource availability to the plant, such as increasing light availability. Second, we consider the effect of changing nutrient requirements of the plant, such as increased nitrogen requirement during a period of seed maturation or luxury consumption. When the plant is exposed to higher light (an exogenous increase from $C_{\max }^{\text {low }}$ to $C_{\max }^{\text {high }}$ ), the trading possibility boundary shifts up at each point by a constant amount $\left(C_{\max }^{\text {high }}-C_{\max }^{\text {low }}\right)$ but the slope at any $N_{\mathrm{G}}$ remains unchanged (compare Fig. 1d to 1c). As a result, the point of intersection of the trading possibility boundary and the stoichiometric ray shifts to the right, indicating that the plant acquires more nitrogen. If every exchange function is convex, then in brighter light the plant utilizes existing trading partners more intensively and may add new partners. To illustrate, the trading possibility boundary in Fig. 1c and 1d is derived from the three exchange functions in Fig. 3. The plant utilizes
$F_{1}$ and $F_{2}$ in low light, but all three fungi in high light. On the other hand, suppose the plant has available not only convex fungi but also one or more concave fungi. Then if the various concave exchange functions are replaced by their lower envelope, the procedure in Fig. 3 can be utilized to derive the trading possibility boundary. Since this "pseudo exchange function" (the lower envelope) has a decreasing marginal-cost curve, additional nitrogen is obtained in brighter light by lowering the common marginal cost, relying less intensively on each of the convex fungi (possibly dumping some) and relying more intensively on one concave fungus (whichever one is activated in the chosen segment of the envelope).

An increase in the plant's nitrogen requirement, relative to its carbon requirement would lead to a decrease in the slope of the stoichiometric ray $\theta$, which would in turn lead to a shift of to the right of the point of intersection of that ray with the trading possibility boundary. The changes to the fungal utilization resulting from this increase in nitrogen demand are analogous to the case of increased light.

\section{Generalizations}

To generalize our model, we relax three of its assumptions. First we relax the assumption that the active mutualist's growth requires combining both resources in a specific ratio. While appropriate for the mycorrhizal mutualism, this assumption may be inappropriate in applications to other mutualisms. Instead, it may be more plausible to assume that growth increases if the active mutualist secures more of one factor and no less of the other factor (regardless of their ratio). Given any new "objective function" of this form the best choice for each active mutualist would continue to occur on the boundary of the trading possibility boundary, and thus our predictions that an active host mutualist equates marginal costs among its trading partners continues to hold as does the prediction that every available mutualist avoided as a trading partner has a higher initial marginal cost. For a more general discussion of why some qualitative properties persist in optimization models of this kind when the objective function is altered, see Salant et al. (1995). The remainder of our qualitative predictions continue to hold if and only if the plant responds to an increase in nitrogen demand by increasing its aggregate acquisition of nitrogen. A condition on the growth function sufficient for this is that nitrogen is what economists term a "normal good" (Varian 1992). Growth functions which do not satisfy this condition are unusual.

Second, we relax the assumption that the nitrogen acquired and carbon retained were used only to maximize the growth of the active mutualist. In a biologically more realistic case, some carbon and nitrogen may be used for defense or reproduction or stored for these uses in the future. However, as long as fitness is increasing with increasing availability of carbon and nitrogen, a 
fitness-maximizing plant will still choose some point on the boundary of the trading possibility boundary and our most basic prediction - equalization of marginal cost — will continue to hold (Salant et al. 1995).

Third, we relax the assumption that the mutualistic partners (represented in our model by the mycorrhizal fungi) do not interact with each other. The assumption of noninteracting fungi may not be biologically realistic because fungi can interact with one another in the soil (Wu et al. 1999) and can possibly exclude one another from the proximity of a potential plant host. It is also possible that fungi may choose to colonize some plants and not others because the fungi themselves can control some physiological and developmental aspects of colonization (Smith and Read 1997).

These mechanisms could impact our model in two ways. Fungal-fungal interactions and the fungal choice of which plant to colonize could restrict the pool of fungi available to the plant. In this case, the plant should still utilize the fungal community with which it is presented in order to maximize its fitness. Accordingly, the plant should still obtain nitrogen from the available fungi to the point where marginal costs are equalized. Therefore, in this more complex situation, our model can still be used to predict the extent of the utilization of each fungus, taking the fungal availability as given. However, our predictions about fungal composition would have to take careful account of which exchange functions are in fact available for the plant to utilize.

It is also possible that the fungus-fungus interactions may alter the nutrient uptake and transfer capabilities of the fungi and thus the fungal interactions may alter the exchange functions associated with the fungi. A thorough analysis of this case is beyond the scope of this paper. However, the analysis would have two parts: (1) the endogenous determination of the exchange functions of potential trading partners and (2) the utilization of these partners by the plant. While the first part would require additional analysis, our analysis does illuminate the second part: given the endogenously determined exchange functions, a fitness-maximizing plant should still equate marginal cost of trading with its fungal partners.

\section{Discussion}

We have shown that partner choice, via differential partner utilization, can lead to coexistence of several mutualistic partners on a single host if the host can trade with the partners on terms of equal marginal cost, and if the sum of the slopes of the marginal cost functions is not negative. These conditions do not hold for partners that have linear or concave exchange functions, and thus the host is predicted to select a single best partner at each level of resource demand. The identity of the selected fungus is predicted to change with changing resource demand of the host. On the other hand, if the partner mutualists have convex exchange functions, which is a biologically plausible proposition, then the two conditions for coexistence do hold, and the host is predicted to associate with one or several partner mutualists. Furthermore, our model predicts the degree of utilization of each partner. Because the degree of partner utilization could in principle be converted to partner abundance, differential partner utilization can determine community structure of the mutualistic partners. The degree of utilization of mutualistic partners, and thus the composition of the partner community, is predicted to change with changes in resource availability to the host mutualist. With increasing availability of the resource controlled by the host mutualist (i.e., increasing light availability leading to increasing carbon availability for a plant) the host mutualist should intensify its use of previously utilized partners and may begin to utilize less effective partners.

The biological feasibility of our model rests on two major assumptions. First, the mutualism must be based on a mutually beneficial exchange of resources or services; i.e., an increase of payment by resource 1 should result in some predictable increase of supply of resource 2. Recent work of Kytoviita (2005) clearly demonstrated that this is the case for the exchange of $\mathrm{C}$ and $\mathrm{N}$ between birch seedlings and an ectomycorrhizal fungus Paxillus involutus. Kytoviita (2005) defoliated seedlings of birch mycorrhizal with $P$. involutus and measured the $\mathrm{C}$ and $\mathrm{N}$ exchange during three days immediately following the defoliation. The defoliated seedlings transferred significantly less $\mathrm{C}$ to the fungus and received significantly less $\mathrm{N}$ from the fungus than nondefoliated controls. Additionally, Kytoviita (2005) reported a strong positive correlation between the amount of $\mathrm{C}$ transferred from the plant to the fungus and the amount of $\mathrm{N}$ transferred from the fungus to the plant.

Second, the host mutualist has to be capable of optimizing the partner utilization, and optimization of partner utilization has to be advantageous to the host. We are not aware of any direct empirical evidence that would confirm or disconfirm this assumption. However, this assumption is consistent with widely observed patterns of apparently adaptive phenotypic plasticity of plants (reviewed by Callaway et al. 2003), such as optimization of root:shoot ratio with respect to nutrient and light availability, altering shoot and root architecture in response to resource availability and presence or absence of neighboring plants.

Our model's predictions of partner coexistence on a single host, and change in partner composition with changing resource availability to the host are consistent with broad patterns of partner coexistence, and shifts in partner composition found in the field (Stanton 2003). However, the extent to which these patterns are caused by economic as opposed to ecological mechanisms (such as resource competition) remains unclear. 


\section{Empirical testing of our model}

The predictions of our model are directly testable, and tests are currently under way in our laboratory. When several fungal trading partners are used simultaneously, a plant in isolation should acquire just enough nitrogen from each partner to equalize marginal cost. This prediction would persist even if the plant used the $\mathrm{N}$ and $\mathrm{C}$ for some other objective besides growth. Testing this prediction is important because - if we find empirically that marginal costs are not equated-an entire class of models (including ours) must be discarded.

Empirical testing of this prediction involves simultaneous measurement of marginal cost in single-planttwo-fungi microcosms where roots of a single plant are split between two fungal compartments. The measurement of the marginal cost of obtaining nitrogen from source $f$ is based on experimentally elevating the plant's demand for nitrogen by a small but measurable increment and measuring the difference in carbon payment by the plant to source $f$ and the difference in nitrogen acquisition from source $f$ between a control condition and an increased nitrogen-demand condition. The formula for determining marginal cost for each fungus is

$$
M C_{f}=\frac{C_{f, \text { increased light }}-C_{f, \text { control }}}{N_{f, \text { increased light }}-N_{f, \text { control }}} .
$$

Nitrogen demand of a plant can be increased by elevating light availability to a light-limited plant. To test the prediction of equal marginal cost, we have to measure the marginal costs of two fungi simultaneously attached to a single plant. This can be done using a splitroot design and stable tracer isotopes.

Measurement of the $\mathrm{N}$ and $\mathrm{C}$ exchange under several levels of altered $\mathrm{N}$ demand would also provide valuable information about the curvature of the exchange functions. If the plant is trading with two fungi, an increase in the light level will reduce the common marginal cost if one fungus has a concave exchange function but will increase the common marginal cost if both fungi are convex.

\section{Comparison with existing theory}

Our focus on the plant's use of multiple trading partners distinguishes our work from prior economicsbased theory in mycorrhizal ecology (Koide and Elliot 1989, Tinker et al. 1994, Toumi et al. 2001). Schwartz and Hoeksema (1998) also focus on a single plant and fungus; however, since they reinterpret the standard theory of international trade, the generalization of their analysis to multiple fungi has already been worked out in the trade literature. Their predictions differ from ours because of a difference in our assumptions.

In our model, a plant may acquire nitrogen from one fungus at one price (defined as carbon paid per unit of nitrogen acquired) and another fungus at a different price. In contrast, Hoeksema and Schwartz assume that the price of the nutrient is the same no matter how much is exchanged and by which trading partners (in economics, the so-called "law of one price" assumption). This is reflected in Schwartz's and Hoeksema's linear "trade acquisition isocline" with endogenous common slope $T$. If their assumption is valid, then every exchange would take place at the same price; so if the plant draws twice as much nutrient from one fungus as from another, it must pay twice as much carbon. In contrast, our model predicts that the price (carbon paid per unit nitrogen received) should vary among the trading partners while the marginal cost of acquiring nitrogen should remain equal among trading partners. This difference between the two models is directly testable by measuring the price of nitrogen in a one-plant-twofungi microcosm.

\section{ACKNOWLEDGMENTS}

We acknowledge the help of Judith Bronstein, Deborah Goldberg, Jacques Crémer, and Ronald Noë. This research was supported by NSF grant DEB-0108524.

\section{Literature Cited}

Axelrod, R., and W. D. Hamilton. 1981. The evolution of cooperation. Science 211:1390-1396.

Bronstein, J. L. 1994. Our current understanding of mutualisms. Quarterly Review of Biology 69(1):31-51.

Bronstein, J. L., W. G. Wilson, and W. F. Morris. 2003. Ecological dynamics of mutualist/antagonist communities. American Naturalist 162:S24-S39.

Bshary, R. S., and J. L. Bronstein. 2004. Game structures in mutualisms: what can the evidence tell us about the kinds of models we need? Advances in the Study of Behavior 34:59-104.

Bull, J. J., and W. R. Rice. 1991. Distinguishing mechanisms for the evolution of cooperation. Journal of Theoretical Biology 149 1):63-74.

Callaway, R. M., S. C. Pennings, and C. L. Richards. 2003. Phenotypic plasticity and interactions among plants. Ecology 84:1115-1128.

Dawkins R. 1976. The selfish gene. Oxford University Press, New York, New York, USA.

Denison, R. F. 2000. Legume sanctions and the evolution of symbiotic cooperation by rhizobia. American Naturalist 156(6):567-576.

Denison, R. F., C. Bledsoe, M. Kahn, F. O'Gara, E. L. Simms, and L. S. Thomashow. 2003. Cooperation in the rhizosphere and the "free rider" problem. Ecology 84:838-845.

Eshel, I., and L. L. Cavallisforza. 1982. Assortment of encounters and evolution of cooperativeness. Proceedings of the National Academy of Sciences (USA) 79:1331-1335.

Ferriere, R., J. L. Bronstein, S. Rinaldi, R. Law, and M. Gauduchon. 2002. Cheating and the evolutionary stability of mutualisms. Proceedings of the Royal Society of London B 269:773-780.

Fischer, M. K., K. H. Hoffmann, and W. Volkl. 2001. Competition for mutualists in an ant-homopteran interaction mediated by hierarchies of ant attendance. Oikos 92: 531-541.

Hoeksema, J. D., and M. Kummel. 2003. Ecological persistence of the plant-mycorrhizal mutualism: a hypothesis from species coexistence theory. American Naturalist 162:S40-S50.

Janos, D. P. 1985. Mycorrhizal fungi: agents of symptoms of tropical community composition? Pages $98-103$ in R. Molina, editor. Proceedings of the sixth North American conference on mycorrhizae. Forest Research Laboratory, Corvallis, Oregon, USA. 
Janzen, D. H. 1985. The natural history of mutualism. Pages 40-99 in D. H. Boucher, editor. The biology of mutualism. Oxford University Press, New York, New York, USA.

Jones, M. D., D. M. Durall, and P. B. Tinker. 1991. Fluxes of carbon and phosphorus between symbionts in willow ectomycorrhizas. New Phytologist 119:99-106.

Jones, M. D., D. M. Durall, and P. B. Tinker. 1998. Comparison of arbuscular and ectomycorrhizal Eucalyptus coccifera. New Phytologist 140:125-134.

Kiers, T. E., R. A. Rousseau, S. A. West, and R. F. Denison. 2003. Host sanctions and the legume-rhizobium mutualism. Nature 425:78-81.

Knowlton, N., and F. Rohwer. 2003. Multispecies microbial mutualisms on coral reefs: the host as a habitat. American Naturalist 162:S51-S62.

Koide, R., and G. Elliot. 1989. Cost, benefit and efficiency of the vesicular arbuscular mycorrhizal symbiosis. Functional Ecology 3:252-255.

Kytoviita, M. M. 2005. Role of nutrient level and defoliation on symbiotic function: experimental evidence by tracing C-14/ $\mathrm{N}-15$ exchange in mycorrhizal birch seedlings. Mycorrhiza 15(1):65-70.

Noë, R. 1990. A veto game played by baboons - a challenge to the use of the prisoners dilemma as a paradigm for reciprocity and cooperation. Animal Behaviour 39:78-90.

Noë, R., and P. Hammerstein. 1994. Biological markets: supply and demand determine the effect of partner choice in cooperation, mutualism, and mating. Behavioral Ecology and Sociobiology 35:1-11.

Sachs, J. L., U. G. Mueller, T. P. Wilcox, and J. J. Bull. 2004. The evolution of cooperation. Quarterly Review of Biology 79(2):135-160.
Salant, S. W., K. L. Kalat, and A. Wheatcroft. 1995. Deducing implications of fitness maximization when a trade-off exists among alternative currencies. Behavioral Ecology 6:424-434.

Schwartz, M. W., and J. D. Hoeksema. 1998. Specialization and resource trade: biological markets as a model of mutualisms. Ecology 79:1029-1038.

Smith, S. E., and D. Read. 1997. Mycorrhizal symbiosis. Academic Press, New York, New York, USA.

Stanton, M. L. 2003. Interacting guilds: moving beyond the pair-wise perspective on mutualisms. American Naturalist 162:S10-S23.

Tinker, P. B., D. M. Durall, and M. D. Jones. 1994. Carbon use efficiency in mycorrhizas: theory and sample calculations. New Phytologist 128:115-122.

Toumi, J., M. M. Kytoviita, and R. Hardling. 2001. Cost efficiency of nutrient acquisition and the advantage of mycorrhizal symbiosis for the host plant. Oikos 92:62-70.

Varian, H. 1992. Microeconomic analysis. W.W. Norton, New York, New York, USA.

Visick, K. L., J. Foster, J. Doino, M. McFall-Ngai, and E. G. Ruby. 2000. Vibrio fischeri lux genes play an important role in colonization and development of the host light organ. Journal of Bacteriology 182(16):4578-4586.

West, S. A., E. T. Kiers, and E. L. Simms. 2002. Sanctions and mutualism stability: why do rhizobia fix nitrogen? Proceedings of the Royal Society of London Series B-Biological Sciences. 269:685-694.

Wu, B., K. Nara, and T. Hogetsu. 1999. Competition between ectomycorrhizal fungi colonizing Pinus densiflora. Mycorrhiza 9:151-159. 